Bull. Korean Math. Soc. 49 (2012), No. 6, pp. 1275-1289

http://dx.doi.org/10.4134/BKMS.2012.49.6.1275

\title{
ISOMETRIC REFLECTIONS IN TWO DIMENSIONS AND DUAL Lํ-STRUCTURES
}

\author{
Francisco J. García-Pacheco
}

\begin{abstract}
In this manuscript we solve in the positive a question informally proposed by Enflo on the measure of the set of isometric reflection vectors in non-Hilbert 2-dimensional real Banach spaces. We also reformulate equivalently the separable quotient problem in terms of isometric reflection vectors. Finally, we give a new and easy example of a real Banach space whose dual has a non-trivial L-summand that does not come from an $M$-ideal in the predual.
\end{abstract}

\section{Isometric reflection vectors in 2-dimensional spaces}

The concept of isometric reflection vector seems to have been officially introduced by Skorik and Zaidenberg in [13].

Definition 1.1 (Skorik and Zaidenberg, [13]). Let $X$ be a real Banach space. A vector $e \in X$ with $\|e\|=1$ is said to be an isometric reflection vector if there exists a closed, maximal subspace $M$ of $X$ such that $X=\mathbb{R} e \oplus M$ and $\|\lambda e+m\|=\|\lambda e-m\|$ for every $m \in M$ and every $\lambda \in \mathbb{R}$. We will denote by $\rho_{e}: X \longrightarrow X$ the surjective linear isometry so that $\rho_{e}(\lambda e+m)=\lambda e-m$ for every $\lambda \in \mathbb{R}$ and every $m \in M$.

Several results of [13] can be summarized into the following one.

Theorem 1.2 (Skorik and Zaidenberg, [13]). Let $X$ be a real Banach space. The following are equivalent:

(1) $X$ is a Hilbert space.

(2) The set of isometric reflection vectors is dense in the unit sphere.

In [3] the authors generalize the previous theorem as follows.

Theorem 1.3 (Becerra-Guerrero and Rodríguez-Palacios, [3]). Let $X$ be a real Banach space. The following are equivalent:

(1) $X$ is a Hilbert space.

Received April 1, 2011; Revised March 7, 2012.

2010 Mathematics Subject Classification. Primary 46B20, 46C05, 46 B04.

Key words and phrases. isometric reflection vector, $\mathrm{L}^{1}{ }_{-}$summand vector.

(C)2012 The Korean Mathematical Society 
(2) The set of the isometric reflection vectors has non-empty interior in the unit sphere.

In [2] it is provided a shorter proof of the previous result. The key of this shortening relies on restricting to 2-dimensions. In Fall 2004, when the author of this manuscript was a graduate student at the Math Department of Kent State University, Per Enflo and the author had a conversation about this shortening, moment at which Enflo asked the author the following question:

Question 1.4. Let $X$ be a finite dimensional real Banach space. Consider on the unit sphere of $X$ a Borel probability measure $\mu$ such that $\mu(\{x\})=0$ for all $x \in X$ such that $\|x\|=1$. Assume that the set of isometric reflection vectors has positive $\mu$-measure. Is then $X$ a Hilbert space?

We would like to let the reader notice that the set of isometric reflection vectors is closed, and hence it is a Borel subset of the unit sphere. Therefore, the previous questions is well formulated. Before presenting any results on the previous question we would like to remark a couple of things.

Remark 1.5. Given a real Banach space we will let $\mathrm{B}_{X}(x, r)$ denote the (closed) ball of center $x$ and radius $r$. As expected, $\mathrm{B}_{X}$ and $\mathrm{S}_{X}$ will denote the (closed) unit ball and the unit sphere of $X$, respectively. On the other hand, let $\|\cdot\|_{0}$ and $\|\cdot\|_{1}$ be two equivalent norms on $X$. The map

$$
\begin{aligned}
\mathrm{S}_{\left(X,\|\cdot\|_{0}\right)} & \rightarrow \mathrm{S}_{\left(X,\|\cdot\|_{1}\right)} \\
x & \mapsto \frac{x}{\|x\|_{1}}
\end{aligned}
$$

is an homeomorphism.

Next, we will show an example, on any finite dimensional real Banach space $X$, of a Borel probability measure $\mu$ on $\mathrm{S}_{X}$ such that $\mu(\{x\})=0$ for all $x \in \mathrm{S}_{X}$.

Example 1.6. Let $X$ be an $n$-dimensional real Banach space. Choose an isomorphism $\psi: \mathbb{R}^{n} \rightarrow X$. This induces an homeomorphism between the unit sphere $\mathrm{S}^{n-1}$ in $\mathbb{R}^{n}$ and $\mathrm{S}_{X}$ as follows:

$$
\begin{aligned}
\phi: \mathrm{S}^{n-1} & \rightarrow \mathrm{S}_{X} \\
x & \mapsto \phi(x):=\frac{\psi(x)}{\|\psi(x)\|} .
\end{aligned}
$$

This homeomorphism also defines a Borel probability measure on $S_{X}$ as follows:

$$
\mu(A):=\mu_{\mathrm{S}^{n-1}}\left(\phi^{-1}(A)\right),
$$

where $A$ is a Borel subset of $\mathrm{S}_{X}$ and $\mu_{\mathrm{S}^{n-1}}$ is the standard measure on $\mathrm{S}^{n-1}$. Notice that $\mu$ depends on the choice of $\psi$.

As a partial positive answer to Question 1.4 we will prove the following:

Theorem 1.7 (Isometric Reflection Dichotomy Theorem). Let $X$ be a 2dimensional real Banach space. Only one of the following two possibilities holds: 
(1) $X$ is a Hilbert space.

(2) The set of isometric reflection vectors is empty or finite.

Before moving into the details of the proof of the previous result we will need the following lemma.

Lemma 1.8. Let $X$ be a 2-dimensional real Banach space $X$. Let $a, b \in \mathrm{S}_{X}$ with $a \neq \pm b$. Denote

$$
\lfloor a, b\rfloor:=\left\{\frac{t a+(1-t) b}{\|t a+(1-t) b\|}: t \in[0,1]\right\} .
$$

Then

(1) $\lfloor a, b\rfloor \subseteq \mathrm{B}_{X}(a,\|a-b\|)$.

(2) If $f \in X^{*}$ is the unique functional such that $f(a)=f(b)=1$, then

$$
\lfloor a, b\rfloor \subseteq f^{-1}[1,+\infty) \cap \mathrm{S}_{X}
$$

(3) The equality holds above if and only if $[a, b]$ is a maximal segment in $\mathrm{B}_{X}$.

Assume, in addition, that $b$ is an isometric reflection vector. Then

(4) $\rho_{b}(\lfloor a, b\rfloor)=\left\lfloor b, \rho_{b}(a)\right\rfloor$.

(5) $\lfloor a, b\rfloor \cap\left\lfloor b, \rho_{b}(a)\right\rfloor=\{b\}$.

(6) $\lfloor a, b\rfloor \cup\left\lfloor b, \rho_{b}(a)\right\rfloor$ is connected.

(7) If $\|a-b\|<1$, then $\lfloor a, b\rfloor \cup\left\lfloor b, \rho_{b}(a)\right\rfloor=\left\lfloor a, \rho_{b}(a)\right\rfloor$.

Proof.

(1) It is a direct consequence of [7, Lemma 3.1].

(2) For any $t \in[0,1]$ we have that $\|t a+(1-t) b\| \leq 1$, therefore

$$
f\left(\frac{t a+(1-t) b}{\|t a+(1-t) b\|}\right)=\frac{1}{\|t a+(1-t) b\|} \geq 1 .
$$

(3) Assume that

$$
\lfloor a, b\rfloor=f^{-1}[1,+\infty) \cap \mathrm{S}_{X} .
$$

If $[a, b]$ is not a maximal segment in $\mathrm{B}_{X}$, then there exists a number $t \in$ $(-\infty, 0) \cup(1, \infty)$ such that $t a+(1-t) b \in \mathrm{S}_{X}$. Obviously, $t a+(1-t) b \in$ $f^{-1}[1,+\infty) \cap \mathrm{S}_{X}$, but $t a+(1-t) b \notin\lfloor a, b\rfloor$. Conversely, assume that $[a, b]$ is a maximal segment in $\mathrm{B}_{X}$. Let $x \in f^{-1}[1,+\infty) \cap \mathrm{S}_{X} \backslash\lfloor a, b\rfloor$. The convexity of $\mathrm{B}_{X}$ forces $a, b$, and $x$ to be aligned, therefore the segment containing them is in the unit sphere and hence $[a, b]$ cannot be maximal.

Assume that $b$ is an isometric reflection vector:

(4) If $t \in[0,1]$, then

$$
\rho_{b}\left(\frac{t a+(1-t) b}{\|t a+(1-t) b\|}\right)=\frac{t \rho_{b}(a)+(1-t) b}{\left\|t \rho_{b}(a)+(1-t) b\right\|},
$$

since $\rho_{b}$ is a surjective linear isometry that fixes $b$. 
(5) Write $\rho_{b}(a)=\gamma b+\delta a$ with $\gamma, \delta \in \mathbb{R}$. Notice that, since $a$ and $b$ are linearly independent, we have that both $\delta$ and $\gamma$ are different from 0 . Besides, $a=\gamma b+\delta \rho_{b}(a)$ and hence

$$
\rho_{b}(a)=-\frac{\gamma}{\delta} b+\frac{1}{\delta} a .
$$

As a consequence, $\delta=-1$. Assume that there exist $t, s \in[0,1)$ such that

$$
\frac{t b+(1-t) a}{\|t b+(1-t) a\|}=\frac{s b+(1-s) \rho_{b}(a)}{\left\|s b+(1-s) \rho_{b}(a)\right\|},
$$

Then

$$
\begin{aligned}
\rho_{b}(a)= & \frac{\|s b+(1-s) a\|(1-t)}{(1-s)\|t b+(1-t) a\|} a \\
& +\frac{\|s b+(1-s) a\|}{(1-s)}\left(\frac{t}{\|t b+(1-t) a\|}-\frac{s}{\|s b+(1-s) a\|}\right) b .
\end{aligned}
$$

Therefore

$$
\frac{\|s b+(1-s) a\|(1-t)}{(1-s)\|t b+(1-t) a\|}=-1,
$$

which is impossible.

(6) $\lfloor a, b\rfloor \cup\left\lfloor b, \rho_{b}(a)\right\rfloor$ is the union of two connected sets whose intersection is non-empty.

(7) If $\|a-b\|<1$, then $\left\|a-\rho_{b}(a)\right\|<2$. Then $\lfloor a, b\rfloor \cup\left\lfloor b, \rho_{b}(a)\right\rfloor$ is a connected set in $S_{X}$ joining $a$ and $\rho_{b}(a)$ and not containing $-a$ or $-\rho_{b}(a)$. Thus $\lfloor a, b\rfloor \cup\left\lfloor b, \rho_{b}(a)\right\rfloor=\left\lfloor a, \rho_{b}(a)\right\rfloor$.

In virtue of the previous lemma we have the following short, but not less important, remark. We spare the details of the proof to the reader.

Remark 1.9. Let $X$ be a 2-dimensional real Banach space. Let $e_{0}, e_{1} \in \mathrm{S}_{X}$ be isometric reflection vectors. We define the following recursive sequence:

$$
e_{n}:=\rho_{e_{n-1}}\left(e_{n-2}\right) \text { for all } n \geq 2 .
$$

Notice the following:

- For every $n \geq 2$ we clearly have that $e_{n}$ is an isometric reflection vector of $X$. Indeed, simply take into consideration that $\rho_{e_{n-1}}$ is a surjective linear isometry on $X$ for $n \geq 2$, and surjective linear isometries map isometric reflection vectors into isometric reflection vectors.

- For every $n \geq 1$ it is clear that

$$
\begin{aligned}
\left\|e_{n}-e_{n-1}\right\| & =\left\|\rho_{e_{n-1}}\left(e_{n-2}\right)-\rho_{e_{n-1}}\left(e_{n-1}\right)\right\| \\
& =\left\|e_{n-1}-e_{n-2}\right\| \\
& =\cdots \\
& =\left\|e_{1}-e_{0}\right\| .
\end{aligned}
$$


- If $e_{0} \neq \pm e_{1}$, then it is also clear, in virtue of the previous item and the fifth item of Lemma 1.8, that

$$
\mathrm{S}_{X}=\bigcup_{n \geq 0}\left\lfloor e_{n}, e_{n+1}\right\rfloor \subseteq \bigcup_{n \geq 0} \mathrm{~B}_{X}\left(e_{n},\left\|e_{1}-e_{0}\right\|\right)
$$

- The sequence $\left(e_{n}\right)_{n \in \mathbb{N}}$ might be a periodic sequence. For instance:

(1) If $e_{0}=e_{1}$, then $e_{n}=e_{0}$ for all $n \geq 0$.

(2) If $e_{0}=-e_{1}$, then $e_{n}=e_{0}$ if $n$ is even and $e_{n}=-e_{0}$ if $n$ is odd.

(3) If $e_{0}$ and $e_{1}$ are in isometric reflection, that is, $X=\mathbb{R} e_{0} \oplus \mathbb{R} e_{1}$ and $\left\|\lambda e_{0}+\gamma e_{1}\right\|=\left\|\lambda e_{0}-\gamma e_{1}\right\|$ for all $\lambda, \gamma \in \mathbb{R}$, then

$$
e_{2}=-e_{0}, e_{3}=-e_{1}, e_{4}=e_{0}, \ldots
$$

Observe that in this case we have that

$$
\mathrm{S}_{X}=\left\lfloor e_{0}, e_{1}\right\rfloor \cup\left\lfloor e_{1}, e_{2}\right\rfloor \cup\left\lfloor e_{2}, e_{3}\right\rfloor \cup\left\lfloor e_{3}, e_{4}\right\rfloor
$$

simply because $e_{0} \neq \pm e_{1}$.

Now we are ready to prove the Isometric Reflection Dichotomy Theorem:

Proof of Theorem 1.7. Assume that there is an infinite sequence $\left(a_{n}\right)_{n \in \mathbb{N}} \subset \mathrm{S}_{X}$ of isometric reflection vectors. Since $S_{X}$ is compact, we can assume that the previous sequence is actually convergent. Our aim is at proving that the set of isometric reflection vectors is dense in $S_{X}$, which will be sufficient in virtue of either [13] or [3]. Fix an arbitrary $0<\varepsilon<1$. There are $n, m \in \mathbb{N}$ such that $\left\|a_{n}-a_{m}\right\| \leq \varepsilon$. We may rename $a_{n}$ and $a_{m}$ as $e_{0}$ and $e_{1}$ respectively. According to Remark 1.9, there exists a sequence of isometric reflection vectors $\left(e_{n}\right)_{n \geq 0} \subset \mathrm{S}_{X}$ such that $\left\|e_{n}-e_{n-1}\right\| \leq \varepsilon$ for all $n \in \mathbb{N}$ and

$$
\mathrm{S}_{X} \subset \bigcup_{n \geq 0} \mathrm{~B}_{X}\left(e_{n}, \varepsilon\right) .
$$

Since $\varepsilon$ was arbitrarily fixed, we conclude that the set of isometric reflection vectors is dense in $S_{X}$.

Corollary 1.10. Let $X$ be a 2-dimensional real Banach space. Let $\mu$ be a Borel probability measure on $\mathrm{S}_{X}$ such that $\mu(\{x\})=0$ for all $x \in \mathrm{S}_{X}$. If the set of isometric reflection vectors has positive $\mu$-measure, then $X$ is a Hilbert space.

Remark 1.11. The Isometric Reflection Dichotomy Theorem fails in any dimension strictly higher than 2. Indeed, let $X$ be any real Banach space of dimension strictly higher than 2 . We will equivalently renorm $X$ to fail the Isometric Reflection Dichotomy Theorem. It is easy to understand that $X$ is isomorphic to a space of the form $\ell_{2}^{2} \oplus_{\infty} M$, where $M$ is a non-zero real Banach space. Now observe that the unit sphere of $\ell_{2}^{2} \oplus_{\infty} M$ has infinitely many isometric reflection vectors but $\ell_{2}^{2} \oplus_{\infty} M$ is not a Hilbert space. 


\section{Applications to the separable quotient problem}

The separable quotient problem is an old and famous problem originally stated by Pelcynzki. However, it was Rosenthal who was the first one to state it in its best known form (see [11]). We refer the reader to [10] for a recopilation of recent results about this problem.

Problem 2.1 (Rosenthal, [11]). Let $X$ be an infinite dimensional, real Banach space. Does $X$ admit an infinite dimensional, separable quotient?

The main result in this section is strongly motivated by the following equivalent form of the separable quotient problem (see [12]). We first remind the reader a couple of things:

- A barrel in a real topological vector space is a closed, absolutely convex subset.

- A real topological vector space is said to be barrelled if every barrel has non-empty interior, or equivalently, is a neighborhood of 0 .

Theorem 2.2 (Saxon and Wilanski, [12]). Let $X$ be an infinite dimensional, real Banach space. The following conditions are equivalent:

(1) $X$ admits an infinite dimensional, separable quotient.

(2) There exists a non-barrelled, dense subspace $Y$ of $X$.

The idea is to reformulate the previous theorem in terms of isometric reflection vectors. Notice that the previous result states that if an infinite dimensional Banach space $X$ admits an infinite dimensional separable quotient, then $X$ has a non-barreled dense subspace $Y$, that is, a dense subspace $Y$ with a barrel that has empty interior in $Y$. We will go a little further on this and we will show that if $X$ has an infinite dimensional separable quotient, then $X$ has a dense subspace $Y$ with a bounded complete barrel that has empty interior in $Y$. For this we will make use of the concept of Markushevich basis: A pair $\left[\left(e_{i}\right)_{i \in I} \subset X,\left(e_{i}^{*}\right)_{i \in I} \subset X^{*}\right]$ is said to be a Markushevich basis when it is biorthogonal $\left(e_{i}^{*}\left(e_{j}\right)=\delta_{i j}\right.$ for all $\left.i, j \in I\right)$, total $\left(\operatorname{span}\left\{e_{i}^{*}: i \in I\right\}\right.$ is $\omega^{*}$-dense in $\left.X^{*}\right)$, and fundamental $\left(\operatorname{span}\left\{e_{i}: i \in I\right\}\right.$ is dense in $\left.X\right)$.

Lemma 2.3. Let $X$ be an infinite dimensional, separable, real Banach space. Let $\left[\left(e_{n}\right)_{n \in \mathbb{N}} \subset \mathrm{S}_{X},\left(e_{n}^{*}\right)_{n \in \mathbb{N}} \subset X^{*}\right]$ be a Markushevich basis for $X$. The linear operator

$$
\begin{aligned}
\ell_{1} & \longrightarrow X \\
\left(t_{n}\right)_{n \in \mathbb{N}} & \longmapsto \sum_{n=1}^{\infty} t_{n} e_{n}
\end{aligned}
$$

maps $\omega^{*}$-closed, bounded subsets of $\ell_{1}$ to sequentially $\omega$-closed subsets of $X$. As a consequence, the set

$$
\left\{\sum_{n=1}^{\infty} t_{n} e_{n}:\left(t_{n}\right)_{n \in \mathbb{N}} \in \mathrm{B}_{\ell_{1}}\right\}
$$


is closed in $X$, and therefore it has empty interior in $X$ if and only if it has empty interior in its linear span.

Proof. Let $A$ be a $\omega^{*}$-closed, bounded subset of $\ell_{1}$. Let $\left(x_{i}\right)_{i \in \mathbb{N}}$ be a sequence in the image of $A$ under the operator (2.1), and assume that $\left(x_{i}\right)_{i \in \mathbb{N}}$ is $\omega$ convergent to some $x_{0} \in X$. For every $i \in \mathbb{N}$, we can write

$$
x_{i}=\sum_{n=1}^{\infty} t_{n}^{i} e_{n}
$$

where $\left(t_{n}^{i}\right)_{n \in \mathbb{N}} \in A$. For every $n \in \mathbb{N}$, we will denote $e_{n}^{*}\left(x_{0}\right)$ by $t_{n}^{0}$. If we fix $n \in \mathbb{N}$, then

$$
\lim _{i \rightarrow \infty} t_{n}^{i}=\lim _{i \rightarrow \infty} e_{n}^{*}\left(x_{i}\right)=e_{n}^{*}\left(x_{0}\right)=t_{n}^{0} .
$$

Since $A$ is bounded, the Banach-Steinhauss Theorem tells us that $\left(t_{n}^{0}\right)_{n \in \mathbb{N}} \in \ell_{1}$ and

$$
\left(t_{n}^{i}\right)_{n \in \mathbb{N}} \stackrel{\omega^{*}}{\longrightarrow}\left(t_{n}^{0}\right)_{n \in \mathbb{N}} \text { as } i \rightarrow \infty .
$$

Since $A$ is $\omega^{*}$-closed we deduce that $\left(t_{n}^{0}\right)_{n \in \mathbb{N}} \in A$. Finally, if $x_{0} \neq \sum_{n=1}^{\infty} t_{n}^{0} e_{n}$, then there is $m \in \mathbb{N}$ such that

$$
t_{m}^{0}=e_{m}^{*}\left(x_{0}\right) \neq e_{m}^{*}\left(\sum_{n=1}^{\infty} t_{n}^{0} e_{n}\right)=t_{m}^{0},
$$

which is impossible.

Lemma 2.4. Let $X$ be an infinite dimensional, separable, real Banach space. Let $\left[\left(e_{n}\right)_{n \in \mathbb{N}} \subset \mathrm{S}_{X},\left(e_{n}^{*}\right)_{n \in \mathbb{N}} \subset X^{*}\right]$ be a Markushevich basis for $X$. The following statements are equivalent:

(1) The basis $\left(e_{n}\right)_{n \in \mathbb{N}}$ is a Schauder basis equivalent to the $\ell_{1}$-basis.

(2) The operator

$$
\begin{aligned}
\ell_{1} & \longrightarrow X \\
\left(t_{n}\right)_{n \in \mathbb{N}} & \longmapsto \sum_{n=1}^{\infty} t_{n} e_{n}
\end{aligned}
$$

is an isomorphism.

(3) The set

$$
\left\{\sum_{n=1}^{\infty} t_{n} e_{n}:\left(t_{n}\right)_{n \in \mathbb{N}} \in \mathrm{B}_{\ell_{1}}\right\}
$$

has non-empty interior.

Proof. Assume that (1) holds. The operator given in (2.3) is onto, therefore it is an isomorphism in accordance to the Open Mapping Theorem. Now, assume that (2) holds. The set given in (2.4) has non-empty interior because it is 
exactly the image under the isomorphism given in (2.3) of the closed unit ball $\mathrm{B}_{\ell_{1}}$. Finally, assume that (3) holds. There exists $r>0$ such that

$$
\mathrm{B}_{X}(0, r) \subseteq\left\{\sum_{n=1}^{\infty} t_{n} e_{n}:\left(t_{n}\right)_{n \in \mathbb{N}} \in \mathrm{B}_{\ell_{1}}\right\} .
$$

If $x \in X \backslash\{0\}$, then

$$
\frac{r x}{\|x\|} \in \mathrm{B}_{X}(0, r) \subseteq\left\{\sum_{n=1}^{\infty} t_{n} e_{n}:\left(t_{n}\right)_{n \in \mathbb{N}} \in \mathrm{B}_{\ell_{1}}\right\} .
$$

Thus, there exists a sequence $\left(s_{n}\right)_{n \in \mathbb{N}} \in \mathrm{B}_{\ell_{1}}$ so that

$$
\frac{r x}{\|x\|}=\sum_{n=1}^{\infty} s_{n} e_{n} \text {. }
$$

In order words,

$$
x=\sum_{n=1}^{\infty} \frac{\|x\| s_{n}}{r} e_{n}
$$

and $\left(\|x\| s_{n} / r\right)_{n \in \mathbb{N}} \in \ell_{1}$.

Lemma 2.5. Let $X$ be an infinite dimensional, separable, real Banach space. There exists a normalized Markushevich basis for $X$ which is not a Schauder basis equivalent to the $\ell_{1}$-basis.

Proof. In virtue of Lemma 2.4, we can assume that $X$ is isomorphic to $\ell_{1}$. Denote by $\left(\mathrm{e}_{n}\right)_{n \in \mathbb{N}}$ the canonical basis of $\ell_{1}$. Consider the sequence $\left(u_{n}\right)_{n \in \mathbb{N}}$ given by $u_{1}=\mathrm{e}_{1}$ and $u_{n}=\left(\mathrm{e}_{n}-\mathrm{e}_{n-1}\right) / 2$ for $n \geq 2$. It is well known that $\left(u_{n}\right)_{n \in \mathbb{N}}$ is a Schauder basis in $\ell_{1}$ (see, for instance, [5]). However,

$$
\sum_{n=1}^{\infty} \frac{1}{n} u_{n}=\frac{3}{4} \mathrm{e}_{1}+\frac{1}{2} \sum_{n=2}^{\infty} \frac{1}{n(n+1)} \mathrm{e}_{n} .
$$

Therefore, $\left(u_{n}\right)_{n \in \mathbb{N}}$ is not equivalent to the $\ell_{1}$-basis.

Lemma 2.6. Let $X$ be an infinite dimensional, real Banach space. If $X$ admits an infinite dimensional, separable quotient, then there exists a proper, dense subspace $E$ of $X$ and a bounded, complete barrel $M$ of $E$ with empty interior in $E$.

Proof. Let $Z$ be a closed subspace of $X$ such that $X / Z$ is an infinite dimensional, separable Banach space. Let $p: X \rightarrow X / Z$ be the canonical projection of $X$ onto $X / Z$. By Lemma 2.5, we can find a bounded sequence $\left(e_{n}\right)_{n \in \mathbb{N}} \subset X$ such that $\left(p\left(e_{n}\right)\right)_{n \in \mathbb{N}}$ is a Markushevich basis for $X / Z$ contained in $\mathrm{S}_{X / Z}$ which is not a Schauder basis equivalent to the $\ell_{1}$-basis. By Lemma 2.3, the set

$$
\left\{\sum_{n=1}^{\infty} t_{n} p\left(e_{n}\right):\left(t_{n}\right)_{n \in \mathbb{N}} \in \mathrm{B}_{\ell_{1}}\right\}
$$


is closed. And, by Lemma 2.4, the above set has empty interior in its linear span. Now, consider

$$
M:=\overline{\mathrm{co}}\left(\left\{\sum_{n=1}^{\infty} t_{n} e_{n}:\left(t_{n}\right)_{n \in \mathbb{N}} \in \mathrm{B}_{\ell_{1}}\right\} \cup \mathrm{B}_{Z}\right)
$$

and $E:=\operatorname{span}(M)$. Note that

$$
M \subseteq p^{-1}\left(\left\{\sum_{n=1}^{\infty} t_{n} p\left(e_{n}\right):\left(t_{n}\right)_{n \in \mathbb{N}} \in \mathrm{B}_{\ell_{1}}\right\}\right) .
$$

Finally, since $p$ is continuous and open, we deduce that $E$ is a dense subspace of $X$ and $M$ is a bounded barrel of $E$ with empty interior in $E$ and closed in $X$.

Observe that, according to Theorem 2.2, the previous lemma is actually a characterization of real Banach spaces admitting an infinite dimensional, separable quotient. Before stating and proving the main result in this section we will make some remarks and reminders.

Remark 2.7. Let $X$ be a real Banach space.

- Assume that $e \in \mathrm{S}_{X}$ is an isometric reflection vector of $e$. The only functional $e^{*} \in X^{*}$ such that $e^{*}(e)=1$ and $\operatorname{ker}\left(e^{*}\right)=\operatorname{ker}\left(\rho_{e}\right)$ is called the isometric reflection functional associated to $e$. In [2] it is shown that $e^{*} \in \mathrm{S}_{X^{*}}$ and that it is an isometric reflection vector of $X^{*}$.

- Consider a vector $e \in \mathrm{S}_{X}$ and a closed, maximal subspace $M$ of $X$ such that $X=\mathbb{R} e \oplus M$. The following conditions are equivalent:

(1) $\|m+\lambda e\|=\|m-\lambda e\|$ for all $\lambda \in \mathbb{R}$ and all $m \in M$.

(2) For all $\lambda \in \mathbb{R}$ and all $m \in M$, if $\lambda e+m \in \mathrm{S}_{X}$, then also $m-\lambda e \in$ $\mathrm{S}_{X}$.

Theorem 2.8. Let $X$ be a real Banach space. Let $e \in \mathrm{S}_{X}$ and $e^{*} \in \mathrm{S}_{X^{*}}$ such that $e^{*}(e)=1$. If $X$ admits an infinite dimensional, separable quotient, then $X$ can be equivalently renormed so that

- $e$ is an isometric reflection vector and $e^{*}$ is the isometric reflection functional associated to $e$.

- $\operatorname{span}\left(\left(\left(e^{*}\right)^{-1}(1) \cap \mathrm{B}_{X}\right)-e\right)$ is a proper dense subspace of $\operatorname{ker}\left(e^{*}\right)$.

Proof. Note that ker $\left(e^{*}\right)$ also has an infinite dimensional, separable quotient. Therefore, by Lemma 2.6, there exists a proper, dense subspace $E$ of $\operatorname{ker}\left(e^{*}\right)$ and a bounded, complete barrel $M$ of $E$ with empty interior in $E$. Observe that we can assume without any loss of generality that $M \subset \mathrm{B}_{\mathrm{ker}\left(e^{*}\right)}$. Now, $X$ can be endowed with the equivalent norm whose unit ball is

$$
\mathcal{B}=\overline{\mathrm{CO}}\left(\mathrm{B}_{\operatorname{ker}\left(e^{*}\right)} \cup(M+e) \cup(M-e)\right) .
$$

(1) The reader may notice that $\mathrm{co}\left(\mathrm{B}_{\mathrm{ker}\left(e^{*}\right)} \cup\{e,-e\}\right)$ has non-empty interior and so does $\mathcal{B}$. Thus, $\mathcal{B}$ is indeed the unit ball of an equivalent norm on $X$. 
(2) $e \in \operatorname{bd}(\mathcal{B})$ (the boundary of $\mathcal{B})$ because $e^{*}(e)=1=\sup e^{*}(\mathcal{B})$.

(3) If $\phi \in X^{*}$, then the linear function $\psi: X \rightarrow \mathbb{R}$ defined as $\psi(v+\lambda e)=$ $\phi(v-\lambda e)$ for all $v \in \operatorname{ker}\left(e^{*}\right)$ and all $\lambda \in \mathbb{R}$ verifies that

$$
\sup \phi(\mathcal{B})=\sup \psi(\mathcal{B}) .
$$

Indeed, it suffices to observe that if $t, s, r \in[0,1]$ with $t+r+s=1$, $m, m^{\prime} \in M$, and $b \in \mathrm{B}_{\operatorname{ker}\left(e^{*}\right)}$, then

$$
t b+s(m+e)+r(m-e), t b+r(m+e)+s(m-e) \in \mathcal{B}
$$

and

$\psi\left(t b+r(m+e)+s\left(m^{\prime}-e\right)\right)=\phi\left(t b+s(m+e)+r\left(m^{\prime}-e\right)\right)$.

(4) $e$ is an isometric reflection vector of $X$ endowed with the $\mathcal{B}$-norm. Indeed, we will rely on Remark 2.7. Let $\gamma \in \mathbb{R}$ and $z \in \operatorname{ker}\left(e^{*}\right)$ such that $\gamma e+z \in \operatorname{bd}(\mathcal{B})$. There exists $\phi \in X^{*} \operatorname{such}$ that $1=\sup \phi(\mathcal{B})=$ $\phi(\gamma e+z)$. Define $\psi: X \rightarrow \mathbb{R}$ as $\psi(v+\lambda e)=\phi(v-\lambda e)$ for all $v \in$ $\operatorname{ker}\left(e^{*}\right)$ and all $\lambda \in \mathbb{R}$. Simply observe that

$$
\sup \psi(\mathcal{B})=1=\phi(\gamma e+z)=\psi(z-\gamma e) .
$$

As a consequence, $z-\gamma e \in \operatorname{bd}(\mathcal{B})$.

(5) $\operatorname{span}\left(\left(\left(e^{*}\right)^{-1}(1) \cap \mathcal{B}\right)-e\right)$ is a proper dense subspace of $\operatorname{ker}\left(e^{*}\right)$. Indeed, it will be enough to show that $\left(\left(e^{*}\right)^{-1}(1) \cap \mathcal{B}\right)-e=M$ or, equivalently, that $\left(e^{*}\right)^{-1}(1) \cap \mathcal{B}=M+e$. On the one hand, we trivially have that $M+e \subseteq\left(e^{*}\right)^{-1}(1) \cap \mathcal{B}$. On the other hand, let $z+e \in\left(e^{*}\right)^{-1}(1) \cap \mathcal{B}$ with $z \in \operatorname{ker}\left(e^{*}\right)$. There exist sequences $\left(t_{n}\right)_{n \in \mathbb{N}},\left(s_{n}\right)_{n \in \mathbb{N}},\left(r_{n}\right)_{n \in \mathbb{N}} \subset$ $[0,1],\left(m_{n}\right)_{n \in \mathbb{N}},\left(m_{n}^{\prime}\right)_{n \in \mathbb{N}} \subset M$, and $\left(b_{n}\right)_{n \in \mathbb{N}} \subset \mathrm{B}_{\text {ker }\left(e^{*}\right)}$ such that $t_{n}+$ $s_{n}+r_{n}=1$ for all $n \in \mathbb{N}$ and $\left(t_{n} b_{n}+s_{n}\left(m_{n}+e\right)+r_{n}\left(m_{n}^{\prime}-e\right)\right)_{n \in \mathbb{N}}$ converges to $z+e$. Because of the product topology, we can deduce (by passing to subsequences if necessary) that $\left(s_{n}\right)_{n \in \mathbb{N}}$ converges to 1 and that $\left(t_{n}\right)_{n \in \mathbb{N}}$ and $\left(r_{n}\right)_{n \in \mathbb{N}}$ both converge to 0 . Therefore, $\left(m_{n}\right)_{n \in \mathbb{N}}$ converges to $z$. Since $M$ is complete, we have that $z \in M$.

Remark 2.9. Observe that given a real Banach space $X$ with an isometric reflection vector $e \in \mathrm{S}_{X}$ and isometric reflection functional $e^{*} \in \mathrm{S}_{X^{*}}$, the set

$$
\left(\left(e^{*}\right)^{-1}(1) \cap \mathrm{B}_{X}\right)-e
$$

is a bounded, complete barrel of its linear span. Indeed, by [6, Theorem 2.2] the above set is absolutely convex, thus it must be absorbing in its linear span. As a consequence, by considering again Theorem 2.2, we have that Theorem 2.8 is in fact a characterization of real Banach spaces admitting an infinite dimensional, separable quotient.

To finish this section, we present the following question (which is actually an equivalent reformulation of the separable quotient problem in virtue of Theorem 2.8 and Remark 2.9). 
Question 2.10. Let $X$ be a real Banach space. Can $X$ be equivalently renormed to have an isometric reflection vector $e \in \mathrm{S}_{X}$ such that

$$
\operatorname{span}\left(\left(\left(e^{*}\right)^{-1}(1) \cap \mathrm{B}_{X}\right)-e\right)
$$

is a proper dense subspace of $\operatorname{ker}\left(e^{*}\right)$ ?

In [6] we presented the following two results that could be considered as an approach to a positive answer to Question 2.10.

Theorem 2.11 (García-Pacheco, [6]). Let $X$ be a real Banach space. Let $e \in \mathrm{S}_{X}$ be an isometric reflection vector. Then

(1) $\overline{\operatorname{span}}\left(\left(\left(e^{*}\right)^{-1}(1) \cap \mathrm{B}_{X}\right)-e\right) \subseteq \operatorname{ker}\left(e^{*}\right)$. Conversely, if $m \in \operatorname{ker}\left(e^{*}\right)$ is so that $\lambda \neq 0$ can be found verifying that $\|e+\lambda m\|=1$, then $m \in$ $\operatorname{span}\left(\left(\left(e^{*}\right)^{-1}(1) \cap \mathrm{B}_{X}\right)-e\right)$.

(2) $\operatorname{span}\left(\left(\left(e^{*}\right)^{-1}(1) \cap \mathrm{B}_{X}\right)-e\right)=\operatorname{ker}\left(e^{*}\right)$ if and only if $\left(e^{*}\right)^{-1}(1) \cap \mathrm{B}_{X}$ is a smooth face of $\mathrm{B}_{X}$.

Theorem 2.12 (García-Pacheco, [6]). Let $X$ be an infinite dimensional, real Banach space. Then $X$ can be equivalently renormed to have an isometric reflection vector $e \in \mathrm{S}_{X}$ such that $\operatorname{span}\left(\left(\left(e^{*}\right)^{-1}(1) \cap \mathrm{B}_{X}\right)-e\right)$ is a closed, maximal subspace of $\operatorname{ker}\left(e^{*}\right)$.

\section{3. $L^{1}$-summand vectors in dual spaces}

A particular case of isometric reflection vectors are the so called $L^{1}$-summand vectors (see [4]).

Definition 3.1 (Behrends, [4]). Let $X$ be a real Banach space. We say that a vector $x \in \mathrm{S}_{X}$ is an $\mathrm{L}^{p}$-summand vector of $X(1 \leq p \leq \infty)$ if there exists a closed maximal subspace $M$ of $X$ such that $X=\mathbb{R} x \oplus_{p} M$.

Combining results from [1] and [6] we have the following theorem, that expresses the differences between $\mathrm{L}^{1}$-summand vectors and isometric reflection vectors in 2-dimensions and in higher dimensions as well.

Theorem 3.2 (Aizpuru and García-Pacheco, [1]). Let L be an uncountably infinite discrete topological space. Denote by $\widehat{L}$ the one-point compactification of $L$ and by $\mathbf{1} \in \mathcal{C}(\widehat{L})$ the constant function on $\widehat{L}$ equal to 1 . Then

(1) If $Y$ is a 2-dimensional subspace of $\mathcal{C}(\widehat{L})$ containing $\mathbf{1}$, then $\mathbf{1}$ is an $\mathrm{L}^{1}$-summand vector of $Y$, and thus it is an isometric reflection vector of $Y$ and a strongly exposed point of $\mathrm{B}_{Y}$.

(2) $\mathbf{1}$ is not an exposed point of $\mathrm{B}_{\mathcal{C}(\widehat{L})}$.

(3) 1 is not an isometric reflection vector of $\mathcal{C}(\widehat{L})$.

The aim of this section is actually at dealing with $\mathrm{L}^{1}$-summand vectors in dual spaces. Taking a look at either [4] or [8] one can quickly realize that if 
$1<p \leq \infty$, then an $\mathrm{L}^{p}$-summand vector of a dual space must be a normattaining functional which attains its norm at an $\mathrm{L}^{q}$-summand vector of the predual space, where $q$ is the conjugate exponent of $p$. More generally, if $1<p \leq \infty$, then an $\mathrm{L}^{p}$-summand subspace of a dual Banach space must be $\omega^{*}$ closed and thus it is the dual of an $L^{q}$-summand subspace of the predual space (in [8] $\mathrm{L}^{1}$-summand subspaces are called $\mathrm{L}$-summands and $\mathrm{L}^{\infty}$-summand subspaces are called M-summands). Precisely in [8, Example IV.1.8] it is shown the existence of a Banach space without non-trivial M-ideals such that $X^{*}$ has a non-trivial L-summand. This example is based on the Theory of Banach Algebras. Here we will show an easy way to find $\mathrm{L}^{1}$-summand vectors in dual spaces that are not norm-attaining functionals at $\mathrm{L}^{\infty}$-summand vectors without using techniques of the ring theory or polynomial algebra. We will rely on the following result (see [9]).

Theorem 3.3 (Jameson, [9]). Let $X$ be a non-reflexive real Banach space. Let $x^{* *} \in X^{* *} \backslash X$. Then

$$
\begin{aligned}
& \left\{x^{*} \in \mathrm{B}_{X^{*}}:\left\|x^{*}\right\|+\frac{\left|x^{* *}\left(x^{*}\right)\right|}{\operatorname{dist}\left(x^{* *}, X\right)} \leq 1\right\} \\
\subseteq & \operatorname{cl}_{\omega^{*}}\left(\mathrm{~B}_{\operatorname{ker}\left(x^{* *}\right)}\right) \\
\subseteq & \left\{x^{*} \in \mathrm{B}_{X^{*}}:\left|x^{* *}\left(x^{*}\right)\right| \leq 2 \operatorname{dist}\left(x^{* *}, X\right)\right\} .
\end{aligned}
$$

In addition

(1) If $X=c_{0}$, then

$$
\left\{x^{*} \in \mathrm{B}_{X^{*}}:\left\|x^{*}\right\|+\frac{\left|x^{* *}\left(x^{*}\right)\right|}{\operatorname{dist}\left(x^{* *}, X\right)} \leq 1\right\}=\operatorname{cl}_{\omega^{*}}\left(\mathrm{~B}_{\operatorname{ker}\left(x^{* *}\right)}\right)
$$

for all $x^{* *} \in X^{* *} \backslash X$.

(2) If $X=\ell_{1}$, then

$$
\operatorname{cl}_{\omega^{*}}\left(\mathrm{~B}_{\operatorname{ker}\left(x^{* *}\right)}\right)=\left\{x^{*} \in \mathrm{B}_{X^{*}}:\left|x^{* *}\left(x^{*}\right)\right| \leq 2 \operatorname{dist}\left(x^{* *}, X\right)\right\}
$$

for all $x^{* *} \in X^{* *} \backslash X$.

Based upon the previous theorem we find the following definition.

Definition 3.4. Let $X$ be a non-reflexive real Banach space. Then

(1) We say that $X$ is of $c_{0}$ dual type if

$$
\left\{x^{*} \in \mathrm{B}_{X^{*}}:\left\|x^{*}\right\|+\frac{\left|x^{* *}\left(x^{*}\right)\right|}{\operatorname{dist}\left(x^{* *}, X\right)} \leq 1\right\}=\operatorname{cl}_{\omega^{*}}\left(\mathrm{~B}_{\operatorname{ker}\left(x^{* *}\right)}\right)
$$

for all $x^{* *} \in X^{* *} \backslash X$.

(2) We say that $X$ is of $\ell_{1}$ dual type if

$$
\operatorname{cl}_{\omega^{*}}\left(\mathrm{~B}_{\operatorname{ker}\left(x^{* *}\right)}\right)=\left\{x^{*} \in \mathrm{B}_{X^{*}}:\left|x^{* *}\left(x^{*}\right)\right| \leq 2 \operatorname{dist}\left(x^{* *}, X\right)\right\}
$$

for all $x^{* *} \in X^{* *} \backslash X$. 
Utilizing plain techniques of the linear algebra and convex geometry we will show the existence of $\mathrm{L}^{1}$-summand vectors in dual spaces that are not normattaining functionals at $\mathrm{L}^{\infty}$-summand vectors in their predual.

Remark 3.5. Let $X$ be a real Banach space.

- If $\left(x_{i}\right)_{i \in I}$ is a net in $\mathrm{B}_{X}$ and $f \in \mathrm{S}_{X^{*}}$ is a norm-attaining functional such that $\left(\left|f\left(x_{i}\right)\right|\right)_{i \in I}$ is convergent to a number $0 \leq k<1$, then we can find another net $\left(z_{i}\right)_{i \in I} \subseteq \mathrm{B}_{X}$ such that $\left(\left|f\left(z_{i}\right)\right|\right)_{i \in I}$ is convergent to $k,\left|f\left(z_{i}\right)\right| \geq k$ for all $i \in I$, and $\left(x_{i}-z_{i}\right)_{i \in I}$ converges to 0 . Indeed, we may assume that $k>f\left(x_{i}\right)>0$ for all $i \in I$ and then it is sufficient to take

$$
z_{i}:=\frac{k-f\left(x_{i}\right)}{1-f\left(x_{i}\right)} z+\frac{1-k}{1-f\left(x_{i}\right)} x_{i},
$$

for all $i \in I$, where $z$ is any element in $\mathrm{S}_{X}$ such that $f(z)=1$.

- One could easily realize that the situation above still holds if $f$ is not norm-attaining, but it would obviously require a much more elaborate proof.

- However, the situation above does not remain true if we ask $k=1$. Indeed, assume that $X$ is a real rotund Banach space whose unit sphere contains a point $z$ that is not strongly exposed on the unit ball. There are then $f \in S_{X *}$ and a sequence $\left(x_{n}\right)_{n \in \mathbb{N}} \subset S_{X}$ such that $f(z)=1$, $\left(f\left(x_{n}\right)\right)_{n \in \mathbb{N}}$ converges to 1 , but $\left(x_{n}\right)_{n \in \mathbb{N}}$ does not converge to $z$. If $\left(z_{n}\right)_{n \in \mathbb{N}} \subset \mathrm{B}_{X}$ is another sequence such that $\left|f\left(z_{n}\right)\right| \geq 1$ for all $n \in \mathbb{N}$, then for all $n \in \mathbb{N}$ we have that either $z_{n}=z$ or $z_{n}=-z$, and hence $\left(x_{n}-z_{n}\right)_{n \in \mathbb{N}}$ cannot converge to 0 .

Remark 3.6. Let $X$ be a non-reflexive real Banach space. The Bishop-Phelps Theorem allows us to deduce that the set of norm-attaining functionals on $X^{*}$ is dense in $X^{* *}$. Therefore, many $x^{*} \in \mathrm{S}_{X^{*}}$ and $x^{* *} \in \mathrm{S}_{X^{* *}}$ can be found such that $x^{* *}\left(x^{*}\right)=1$ and $0<\operatorname{dist}\left(x^{* *}, X\right) \leq \frac{1}{2}$.

Theorem 3.7. Let $X$ be a non-reflexive real Banach space of $c_{0}$ dual type. Let $x^{*} \in \mathrm{S}_{X^{*}}$ and $x^{* *} \in \mathrm{S}_{X^{* *}}$ such that $x^{* *}\left(x^{*}\right)=1$ and $0<\operatorname{dist}\left(x^{* *}, X\right) \leq \frac{1}{2}$. The equivalent norm on $X^{*}$ given for all $y^{*} \in X^{*}$ by

$$
\left\|y^{*}\right\|_{1}:=\left|x^{* *}\left(y^{*}\right)\right|+\left\|y^{*}-x^{* *}\left(y^{*}\right) x^{*}\right\|
$$

is a dual norm.

Proof. Let $\left(y_{i}^{*}\right)_{i \in I}$ be a net in $X^{*}$ such that $\left\|y_{i}^{*}\right\|_{1} \leq 1$ and assume that $\left(y_{i}^{*}\right)_{i \in I}$ is $\omega^{*}$ convergent to some $y^{*} \in X^{*}$. We may assume that $\left(\left|x^{* *}\left(y_{i}^{*}\right)\right|\right)_{i \in I}$ is convergent to some number $0 \leq k \leq 1$. Observe also that if $k=1$, then $y^{*}=x^{*}$ and thus $\left\|y^{*}\right\|_{1}=1$. So, assume that $k<1$. Notice that in virtue of the previous remark we may assume that $\left|x^{* *}\left(y_{i}^{*}\right)\right| \geq k$ for all $i \in I$. Since $\left\|y_{i}^{*}-x^{* *}\left(y_{i}^{*}\right) x^{*}\right\| \leq 1-\left|x^{* *}\left(y_{i}^{*}\right)\right| \leq 1-k$ for all $i \in I$, we deduce that

$$
\left(\frac{y_{i}^{*}-x^{* *}\left(y_{i}^{*}\right) x^{*}}{1-k}\right)_{i \in I} \subseteq \mathrm{B}_{\operatorname{ker}\left(x^{* *}\right)}
$$


is a net $\omega^{*}$ convergent to

$$
\frac{y^{*}-k x^{*}}{1-k}
$$

By hypothesis,

$$
\left\|\frac{y^{*}-k x^{*}}{1-k}\right\|+2 \frac{\left|x^{* *}\left(y^{*}\right)-k\right|}{1-k} \leq 1 .
$$

In other words,

$$
\begin{aligned}
\left\|y^{*}\right\|_{1} & =\left|x^{* *}\left(y^{*}\right)\right|+\left\|y^{*}-x^{* *}\left(y^{*}\right) x^{*}\right\| \\
& \leq\left\|y^{*}-k x^{*}\right\|+2\left|x^{* *}\left(y^{*}\right)-k\right|+k \\
& \leq 1
\end{aligned}
$$

Corollary 3.8. Let $X$ be a non-reflexive real Banach space of $c_{0}$ dual type. Then $X$ can be equivalently renormed so that its dual has an $\mathrm{L}^{1}$-summand vector that is not a norm-attaining functional at an $\mathrm{L}^{\infty}$-summand vector in $X$.

To finish the manuscript we will show that the hypothesis of $c_{0}$ dual type in the previous result is necessary.

Remark 3.9. Let $X$ be a real Banach space. Consider $a \neq b \in X \backslash\{0\}$ and assume that $\|a\|>\|b\|$. The triangular inequality tells us that $\|a\|-\|b\| \leq$ $\|a-b\|$. Take a look at the following:

$$
\frac{a}{\|a\|}=\frac{\|b\|}{\|a\|} \frac{b}{\|b\|}+\frac{\|a\|-\|b\|}{\|a\|} \frac{a-b}{\|a\|-\|b\|} .
$$

If $\|a\|-\|b\|=\|a-b\|$, then we deduce that

$$
\frac{a}{\|a\|} \in\left[\frac{b}{\|b\|}, \frac{a-b}{\|a-b\|}\right] \subset \mathrm{S}_{X} .
$$

Theorem 3.10. Let $X$ be a non-reflexive real Banach space of $\ell_{1}$ dual type. Let $x^{*} \in \mathrm{S}_{X^{*}}$ and $x^{* *} \in \mathrm{S}_{X^{* *}} \backslash X$ such that $x^{* *}\left(x^{*}\right)=1$. Consider the equivalent norm on $X^{*}$ given by

$$
\left\|y^{*}\right\|_{1}:=\left|x^{* *}\left(y^{*}\right)\right|+\left\|y^{*}-x^{* *}\left(y^{*}\right) x^{*}\right\|
$$

for all $y^{*} \in X^{*}$. If $x^{*}$ is a rotund point of $\mathrm{B}_{X^{*}}$, i.e. $x^{*}$ does not belong to any non-trivial segment of $\mathrm{S}_{X^{*}}$, then $\|\cdot\|_{1}$ is not a dual norm on $X^{*}$.

Proof. Take $y^{*} \in \mathrm{S}_{X *}$ such that

$$
0<x^{* *}\left(y^{*}\right)<\min \left\{1,2 \operatorname{dist}\left(x^{* *}, X\right)\right\} .
$$

By hypothesis, $y^{*} \in \operatorname{cl}_{\omega^{*}}\left(B_{\operatorname{ker}\left(x^{* *}\right)}\right)$. However, according to the previous remark and by assumption, we have:

$$
\begin{aligned}
\left\|y^{*}\right\|_{1} & =\left|x^{* *}\left(y^{*}\right)\right|+\left\|y^{*}-x^{* *}\left(y^{*}\right) x^{*}\right\| \\
& >\left|x^{* *}\left(y^{*}\right)\right|+\left\|y^{*}\right\|-\left\|x^{* *}\left(y^{*}\right) x^{*}\right\| \\
& =1 .
\end{aligned}
$$

Therefore, $\|\cdot\|_{1}$ is not a dual norm on $X^{*}$. 


\section{References}

[1] A. Aizpuru and F. J. García-Pacheco, A short note about exposed points in real Banach spaces, Acta Math. Sci. Ser. B Engl. Ed. 28 (2008), no. 4, 797-800.

[2] A. Aizpuru, F. J. García-Pacheco, and F. Rambla, Isometric reflection vectors in Banach spaces, J. Math. Anal. Appl. 299 (2004), no. 1, 40-48.

[3] J. Becerra-Guerrero and A. Rodríguez-Palacios, Isometric reflections on Banach spaces after a paper of A. Skorik and M. Zaidenberg, Rocky Mountain J. Math. 30 (2000), no. 1, 63-83.

[4] E. Behrends et al., $\mathrm{L}_{p}$-Structure in Real Banach Spaces, Lecture Notes in Mathematics, 613, Springer-Verlag, Berlin-Heidelberg-New York, 1977.

[5] J. Diestel, Sequences and Series in Banach Spaces, Springer-Verlag, New York, 1984.

[6] F. J. García-Pacheco, Geometry of isometric reflection vectors, Math. Slovaca 61 (2010), no. 5, 807-816.

[7] _ Rotundity and connectedness in two dimensions, Bol. Soc. Mat. Mexicana (3) 15 (2009), no. 2, 165-173.

[8] P. Hadamard, D. Werner, and W. Werner, M-Ideals in Banach Spaces and Banach Algebras, Springer-Verlag, Berlin, 1993.

[9] G. J. O. Jameson, The weak star closure of the unit ball in a hyperplane, Proc. Edinburgh Math. Soc. (2) 18 (1972), no. 2, 7-11.

[10] J. Mújica, Separable quotients of Banach spaces, Rev. Mat. Univ. Complut. Madrid 10 (1998), 299-330.

[11] H. P. Rosenthal, On quasi-complemented subspaces of Banach spaces, Proc. Natl. Acad. Sci. USA 59 (1968), 361-364.

[12] S. A. Saxon and A. Wilansky, The equivalence of some Banach space problems, Colloq. Math. 37 (1977), no. 2, 217-226.

[13] A. Skorik and M. Zaidenberg, On isometric reflections in Banach spaces, Mat. Fiz. Anal. Geom. 4 (1997), no. 1-2, 212-247.

Department of Mathematics

TEXAs A\&M UNIVERSITY

College Station, Texas, 77843-3368, USA

E-mail address: fgarcia@math.tamu.edu 